\title{
Trastornos de la conducción en el posoperatorio de cirugía cardíaca
}

\author{
Dres. Zuly Cortellezzi', Gustavo Tortajada¹, Romina Roif1, Gonzalo Varela²
}

\section{Resumen}

Durante el posoperatorio de cirugía cardíaca es frecuente observar trastornos en la generación o conducción del ritmo cardíaco, o en ambos, los cuales en su mayoría son transitorios. Sin embargo, un porcentaje de ellos requerirán implante de marcapasos definitivo. Conocer los predictores preoperatorios que se asocian a implante de marcapasos definitivo (características del paciente, tipo de cirugía, etcétera) nos permitirá adecuar el tratamiento y reducir la morbimortalidad, la estadía hospitalaria y los costos sanitarios durante el posoperatorio.

Palabras clave:

\author{
CIRUGÍA CARDÍACA \\ POSOPERATORIO \\ TRASTORNOS DE LA CONDUCCIÓN \\ IMPLANTE MARCAPASOS DEFINITIVO
}

\section{Conduction disorders in the postoperative period of cardiac surgery}

\section{Summary}

During postoperative care of cardiac surgery heart rhythm disorders are frequently seen. Most of them are transient, but some may need permanent pacemaker implantation. Knowing preoperative permanent pacemaker implantation predictors (i.e. patients characteristics, surgery type, etc.) will allow us to provide a better patient treatment, reduce morbidity and mortality, in-hospital length of stay and health care costs.

Key words:

\author{
CARDIAC SURGERY \\ POSTOPERATIVE \\ HEART CONDUCTION DISORDERS \\ PERMANENT PACEMAKER
}

\section{Distúrbios de condução no pós-operatório de cirurgia cardíaca Resumo \\ Durante o período pós-operatório de cirurgia cardiaca é comum observar distúrbios na geração e/ou condução do ritmo cardíaco, que em sua maioria, são transitórios. No entanto, uma porcentagem deles exigirá o implante do marcapasso de- finitivo. Por este motivo, é fundamental o conhecimento dos preditores pré-operatórios associados à implantação defini- tiva do marcapasso (características do paciente, tipo de cirurgia, etc.) para que se possa melhorar o tratamento e reduzir a morbimortalidade, o tempo de internação e os custos de saúde. \\ Palavras chave: CIRURGIA CARDIACA \\ PÓS-OPERATORIO \\ DISTÚRBIOS DE CONDUÇÃO \\ MARCAPASSO DEFINITIVO}

1. Servicio de Electrofisiología, Centro Cardiovascular, Casa de Galicia. Montevideo, Uruguay.

2. Departamento de Cardiología, Hospital Británico. Montevideo, Uruguay.

Correspondencia: Dra. Zuly Cortellezzi. Correo electrónico: seefarritmias@gmail.com

Los autores declaran no tener conflictos de intereses.

Recibido Oct 5, 2020; aceptado Oct 11, 2020 


\section{Introducción}

Las alteraciones en el sistema de conducción son frecuentes en el posoperatorio de cirugía cardíaca (POCC), presentándose hasta en la mitad de los pacientes. Comprenden diferentes grados de bloqueo auriculoventricular (BAV), bloqueos fasciculares y enfermedad del nodo sinusal (ENS). La mayoría de estos trastornos son transitorios y reversibles. En ocasiones, es necesaria la estimulación temporal mediante electrodos epicárdicos para mantener un adecuado gasto cardíaco.

La necesidad de implante posoperatorio de marcapasos definitivo (MPD) es baja, 1,4\%-3,0\%, variando según el tipo de procedimiento quirúrgico realizado $^{(1-4)}$. La indicación principal para la estimulación permanente es el BAV de alto grado.

Estos trastornos aumentan la morbilidad y prolongan la estadía hospitalaria, pero no se ha demostrado que determinen un aumento de la mortalidad operatoria. Durante la valoración preoperatoria es muy importante conocer la frecuencia de estas complicaciones, según el tipo de cirugía y los factores predisponentes, para el correcto manejo posoperatorio.

\section{Epidemiología}

Los trastornos de la conducción son frecuentes en la cirugía de revascularización miocárdica (CRM), siendo el bloqueo de rama derecha el más común ${ }^{(5)}$. El BAV completo se evidencia en alrededor del $16 \%$ de las cirugías ${ }^{(6-8)}$, pero solo $0,23 \%-1,1 \%$ de los pacientes requieren MPD, según las diferentes series $^{(2-4,8,9)}$. En la cirugía valvular la incidencia es mayor, entre $3,0 \%$ y $6,0 \%{ }^{(2,4,9,10)}$. Para la cirugía combinada se reporta una incidencia de $6,1 \%{ }^{(4)}$.

En el caso de la cirugía de sustitución valvular aórtica (SVA), 2,5\%-6,6\%(3,4,9,11-13) de los pacientes requiere $\mathrm{MPD}$, y esta cifra aumenta a más del doble si se utilizan válvulas sin sutura ${ }^{(15,16)}$.

El 23\% de los pacientes sometidos a reparación o reemplazo de válvula mitral presentan BAV de primer, segundo o tercer grado, requiriendo MPD 2,0\%-4,4\% de ellos ${ }^{(9,17-19)}$.

Debido a la baja frecuencia de la cirugía aislada sobre la válvula tricúspide en adultos, la incidencia en esta intervención por sí sola es difícil de establecer. En un estudio de sustitución valvular tricúspide, por prótesis biológica o mecánica, se informó que la necesidad de implante de MPD fue de $22 \%{ }^{(20)}$. Wiggins y colaboradores ${ }^{(4)}$, por su parte, reportaron una incidencia de $25 \%$ y $12 \%$ para la sustitución y reparación valvular tricúspide, respectivamente.

El riesgo de bloqueo e implante de MPD aumenta con el número de válvulas sustituidas. Según un estudio publicado por Leyva y colaboradores ${ }^{(9)}$, que incluyó 135.356 pacientes, la incidencia de MPD fue de $4,22 \%$ para la SVA, 4,38\% para la mitral, $5,59 \%$ para la cirugía combinada aórtica y mitral y 7,89\% para la mitral y tricúspide. Por su parte, la reintervención valvular determina un aumento significativo en el riesgo de requerir MPD, pudiendo llegar hasta $10,5 \%{ }^{(21-23)}$.

\section{Anatomía del sistema de conducción}

Para comprender los mecanismos implicados en estos trastornos es importante conocer la anatomía del sistema de conducción, especialmente su irrigación y la proximidad con las válvulas cardíacas. El sistema de conducción comprende el nodo sinusal (NS) encargado de la generación, propagación y distribución del impulso eléctrico cardíaco, el nodo auriculoventricular (NAV) que optimiza la transmisión del impulso eléctrico de aurículas a ventrículos, y el sistema His-Purkinje que distribuye el impulso para la contracción ventricular izquierda y derecha. El NS tiene una ubicación subepicárdica a lo largo de la pared ántero-lateral de la aurícula derecha, próximo a la desembocadura de la cava superior. El NAV es una estructura subendocárdica auricular derecha, ubicada en el tabique interauricular, anterior al orificio del seno coronario y encima de la inserción de la lámina septal de la válvula tricúspide, región conocida como triángulo de Koch (figura 1). La unión AV se divide en tres zonas: la región de células transicionales, el nodo AV compacto y el haz penetrante de His. La estructura histológica del NAV es compleja, encontrándose gran variabilidad interindividual, según la edad, la presencia de tejido graso y fibrosis. El haz penetrante de His es la única estructura del sistema de conducción que penetra el cuerpo fibroso central, formado por la unión del tejido conectivo de los velos valvulares aórtico y mitral, y por el velo septal de la válvula tricúspide. Luego atraviesa el septum membranoso interventricular y se divide en sus ramas derecha e izquierda (figura 2). La rama derecha pasa a través de la musculatura del tabique interventricular a la base del músculo papilar medial del ventrículo derecho. La rama izquierda, desde su origen, situado debajo de la comisura entre los velos derecho y no coronario de la válvula aórtica, desciende por el subendocardio del tabique interventricular y se divide en rama anterior y posterior ${ }^{(24,25)}$. La irrigación del NS depende de la arteria coronaria derecha en $73 \%$ de los casos, en $3 \%$ de la coronaria izquierda, y en el $23 \%$ restante de ambas, formando un anillo. La arteria del nodo AV es responsable de la irrigación de dicho nodo y es rama de la coronaria derecha en $80 \%$ de los casos, de 


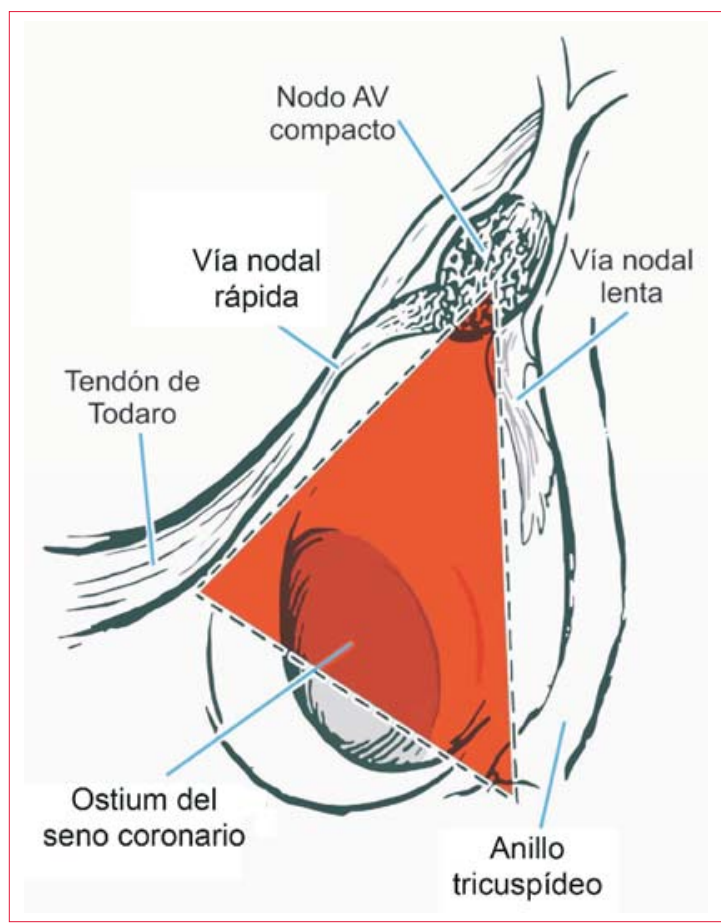

Figura 1. Anatomía del triángulo de Koch. Extraído de Texto de Cardiología, Sociedad Interamericana de Cardiología(25).

la arteria circunfleja en $10 \%$ y de ambas en el $10 \%$ restante. El haz de His es irrigado, en $75 \%$ de los casos, por las ramas perforantes de la arteria coronaria izquierda, por la coronaria derecha en $5 \%-10 \%$, y por ambas arterias en 15\%-20\%. Su sector proximal se abastece a través de la arteria coronaria derecha, pero a medida que se extiende periféricamente, el porcentaje de irrigación desde la arteria coronaria izquierda aumenta gradualmente, siendo los haces derecho e izquierdo, en todos los casos, irrigados por perforantes de la arteria coronaria izquierda ${ }^{(26)}$.

\section{Fisiopatología}

Los mecanismos fisiopatológicos principales que explican las alteraciones de la conducción observadas en el POCC son el traumatismo mecánico directo, la isquemia miocárdica y la inadecuada protección cardíaca.

El trauma mecánico se relaciona principalmente con la cirugía valvular, y es conocido desde hace muchos años que la proximidad del sistema de conducción a las válvulas cardíacas es el factor determinante. En 1935, Yater y colaboradores ${ }^{(27)}$ ya describían el BAV en asociación con calcificación del anillo aórtico, y en 1963, Sellers y colaboradores ${ }^{(28)} \mathrm{de}$ mostraron por primera vez una lesión en el sistema de conducción durante el implante de una válvula mecánica aórtica. En estudios histopatológicos rea-

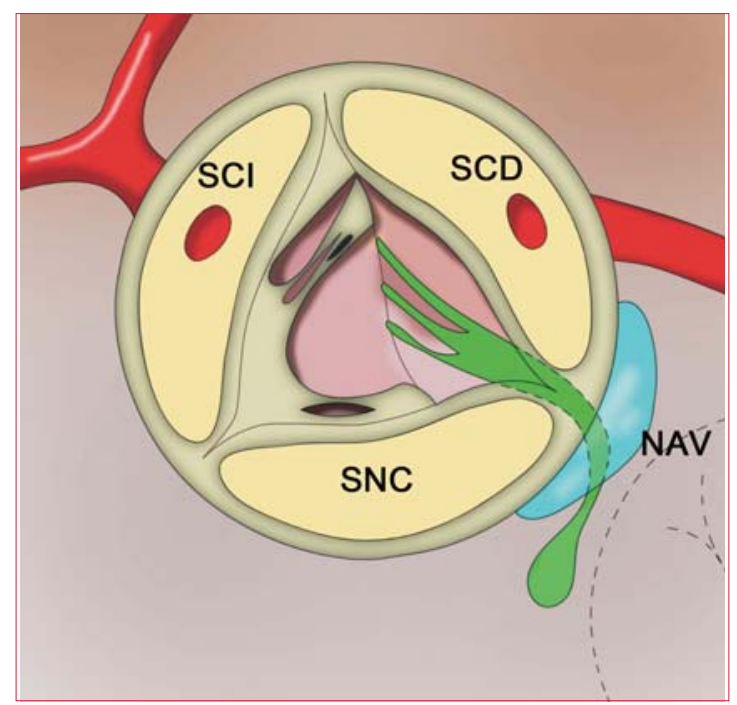

Figura 2. Relación del sistema de conducción con la válvula aórtica. SCI: seno coronariano izquierdo; SCD: seno coronariano derecho; SNC: seno no coronariano; NAV: nodo auriculoventricular.

lizados a pacientes con cirugía valvular aórtica, Fukuda y colaboradores ${ }^{(29)}$ identificaron tres tipos de lesiones morfológicas del sistema de conducción: lesiones compatibles con degeneración crónica y fibrosis (identificadas como factores predisponentes), lesiones no traumáticas, como hemorragia y edema (reversibles en pocos días), y lesiones traumáticas persistentes (relacionadas con la decalcificación anular y lesiones por suturas). Como se describió anteriormente, el área debajo de la unión de las valvas aórticas derecha y no coronariana corresponde a la extremidad anterior del haz de His previo a subdividirse en sus ramas, por lo que cualquier trauma directo o tensión mecánica en esta región podría explicar la aparición de trastornos de la conducción. La lesión puede producirse durante el desbridamiento del calcio valvular o durante la sutura de la válvula, sobre todo en la técnica de sutura continua $^{(30)}$. Por otro lado, cuando la insuficiencia aórtica es la patología predominante, la ectasia anular puede imponer un estiramiento mecánico crónico y progresivo entre el NAV y el haz de His, lo que aumenta el riesgo de daño irreversible en la cirugía(10). El hecho de que la mayoría de los trastornos de la conducción, en particular el BAV competo, ocurran durante o dentro de las primeras horas de la cirugía, respalda el planteo de la lesión o tensión mecánica como principal mecanismo.

La válvula mitral también está anatómicamente próxima al sistema de conducción, especialmente su comisura medial posterior; sin embargo, no hay estudios que evidencien alteraciones morfológicas sobre el sistema de conducción en la cirugía mitral. Berdajs y colaboradores ${ }^{(18)}$ no encontraron predic- 
tores de BAV en pacientes con cirugías de sustitución o anuloplastia mitral y plantearon la hipótesis de que el daño de la arteria del NAV podría desempeñar un papel en su desarrollo. Los autores describieron que en $23 \%$ de los pacientes esta arteria está anatómicamente muy próxima al anillo mitral.

Como vimos, el NAV está íntimamente relacionado con la valva septal de la válvula tricúspide, lo que lo hace particularmente susceptible durante cualquier intervención sobre ésta. Se han desarrollado diferentes técnicas quirúrgicas de reparación valvular, incluyendo la utilización de anillos tricúspides incompletos para disminuir su lesión.

Durante la endocarditis valvular, el BAV puede ocurrir de manera secundaria a la extensión del proceso infeccioso. El bloqueo cardíaco se presenta más frecuentemente en la endocarditis de la válvula aórtica y se asocia con mayor riesgo de muerte súbita cardíaca y destrucción anatómica más severa de los tejidos cardíacos ${ }^{(31)}$.

La isquemia miocárdica y la pobre protección cardíaca son los mecanismos más frecuentemente involucrados en la génesis de trastornos de la conducción durante la CRM, aunque también participan en la cirugía valvular. En una revisión de Kumbhani y colaboradores ${ }^{(5)}$, la isquemia ha sido implicada como el factor más importante por la mayoría de los autores, existiendo una correlación entre el número y grado de estenosis de los vasos coronarios, y el desarrollo y severidad de los trastornos de la conducción. Caspi y colaboradores ${ }^{(7)}$ encontraron que la combinación de enfermedad obstructiva proximal de la arteria coronaria izquierda y oclusión de la arteria coronaria derecha dominante fue más frecuente en pacientes que presentaban BAV que en aquellos que no lo presentaron ( $32 \%$ vs. $12 \%$, $\mathrm{p}<0,05)$. Plantearon que esta diferencia se debía a una insuficiente protección miocárdica por distribución no uniforme de la solución de cardioplejia y a la menor revascularización de la arteria coronaria derecha (y por ende de la arteria del NAV). El infarto de miocardio aumenta la incidencia de BAV por daño isquémico agudo del sistema de conducción $^{(7,8)}$. El tejido de conducción difiere de los miocitos cardíacos por ser menos tolerante a los efectos de la isquemia, hiperpotasemia e hipotermia (estos dos últimos, sistémicos o inducidos por una solución cardiopléjica fría y rica en potasio) ${ }^{(5)}$. Se ha planteado que la protección miocárdica sería mayor con cardioplejia normotérmica y con una distribución más uniforme a través de perfusión anterógrada y retrógrada.

El deterioro en la irrigación coronaria aumenta con la edad, al igual que la frecuencia de enfermeda- des degenerativas del sistema de conducción, lo que aumenta la probabilidad de BAV.

La ENS tiene una incidencia menor que los BAV y fasciculares luego de cirugía cardíaca. Puede expresarse como bradicardia sinusal persistente o ritmos de la unión que requieren estimulación. En su mayoría, se deben a sufrimiento isquémico, aunque también pueden ser secundarios a un trauma producido durante la auriculotomía lateral derecha, $\mathrm{u}$ otros abordajes transeptales superiores que pueden ser utilizados en la cirugía mitral o durante la ablación de fibrilación auricular. La presencia, en algunos pacientes, de un anillo vascular sino-auricular formado por colaterales de ambas arterias coronarias, podría explicar la recuperación del NS al daño isquémico causado por infarto local, incisión quirúrgica o pobre protección miocárdica ${ }^{(26)}$.

En el posoperatorio inmediato es frecuente observar ritmos de la unión acelerados transitorios. Su etiología es multifactorial y generalmente no implican un trastorno de la conducción en sí mismos. Se caracterizan por comandar transitoriamente el ritmo cardíaco, dado que su alta frecuencia inhibe al NS por sobreestimulación.

\section{Predictores de implante de marcapasos definitivo}

Durante la valoración preoperatoria la posibilidad de identificar a los pacientes que necesitarán un MPD luego de la cirugía cardíaca, es baja. Conocer los predictores asociados más importantes nos será útil al momento de informar a nuestro paciente.

Diferentes estudios han definido factores de riesgo relacionados al desarrollo de trastornos de la conducción permanentes en el posoperatorio. En 1984, Goldman y colaboradores ${ }^{(2)}$ ya establecían como predictores de riesgo las alteraciones de la conducción previas, edad avanzada, anillo valvular aórtico calcificado, cirugía valvular (especialmente de la válvula tricúspide) y pobre protección miocárdica. En más de 10.000 pacientes sometidos a cirugía cardíaca, Gordon y colaboradores ${ }^{(1)}$ encontraron cinco factores de riesgo independientes relacionados con el trauma físico del sistema de conducción (cirugía valvular, reintervención, cirugía de ablación de arritmias, reconstrucción del anillo mitral y endocarditis activa), y otros relacionados con un aumento de la carga isquémica (edad $\geq 75$ años, insuficiencia renal y pobre protección miocárdica durante la cirugía). Usando la fórmula de probabilidad propuesta por los autores, se puede calcular el riesgo de MPD para diferentes escenarios, por ejemplo, el riesgo en una CRM sin complicaciones es de 0,9\%, 
mientras que para una cirugía de reintervención valvular aórtica por endocarditis es de $29,4 \%$.

Otro estudio encontró que los trastornos de la conducción previos, especialmente el bloqueo completo de rama izquierda (BCRI), o diferentes grados de BAV y la cirugía de SVA, son predictores independientes de MPD en el POCC ${ }^{(3)}$. En 1.200 cirugías cardíacas, Socie y colaboradores ${ }^{(15)}$ establecieron como predictores de MPD, la edad, sexo femenino, endocarditis infecciosa y cirugía de SVA.

Una revisión publicada en el $2010^{(32)}$, que incluyó 2.557 pacientes con SVA, estableció la enfermedad previa del sistema de conducción como el predictor más potente de BAV posoperatorio. Otros predictores descriptos fueron la insuficiencia aórtica (como patología valvular primaria), válvula aórtica bicúspide, calcificación del anillo aórtico, disfunción ventricular severa, hipertensión arterial, tiempo prolongado de circulación extracorpórea, y la técnica quirúrgica de sutura continua de la válvula. Estudios posteriores ${ }^{(3,14,33,34)}$ también encontraron a los trastornos de la conducción preoperatorios como predictores independientes, cuadruplicando el riesgo en algunos trabajos ${ }^{(14)}$.

En 1978, Friedman y colaboradores ${ }^{(35)}$ demostraron, mediante estudio electrofisiológico, que el $30 \%$ de los pacientes con estenosis aórtica calcificada presentaban alteraciones en la conducción intra o infra His (intervalo HV prolongado), mientras que los pacientes con insuficiencia aórtica crónica mostraban más frecuentemente un intervalo PR y AH prolongados. La prolongación del HV en la enfermedad valvular aórtica calcificada puede no tener expresión en el electrocardiograma (ECG) de superficie o solo manifestarse con un discreto aumento del intervalo PR.

Koplan y colaboradores ${ }^{(36)}$ elaboraron un score simple y útil para predecir el riesgo de MPD luego de la cirugía valvular. Este establece como predictores independientes más fuertes el bloqueo completo de rama derecha (BCRD) (OR: 3,6) y la cirugía multivalvular, que incluye la válvula tricúspide (OR: 3,7 ), a los cuales le adjudica 2 puntos; la cirugía multiválvula sin válvula tricúspide (OR: 2,1), BCRI preoperatorio (OR: 2,0), BAV de primer grado (OR: $1,9)$, cirugía valvular previa (OR: 1,8$)$ y la edad $>70$ años (OR: 1,4) con 1 punto cada uno. Un puntaje de riesgo de 0-6 identificó pacientes en el grupo de validación con incidencias de MPD de 1,9\%, 5,2\%, 8,7\%, $11,5 \%, 21 \%, 36 \%$ y $50 \%$, respectivamente.

Resumiendo, los predictores más importantes para evaluar el riesgo de implante de MPD en el posoperatorio son: 1) la presencia de trastornos de la conducción previos, especialmente bloqueos de rama; 2) la mayor edad del paciente, asociada a mayo- res alteraciones degenerativas en el sistema de conducción; 3) la cirugía valvular, destacando la cirugía valvular aórtica con extensa calcificación anular, la cirugía por endocarditis valvular complicada con abscesos y la cirugía de múltiples válvulas que incluye a la válvula tricúspide; 4) la cirugía de reintervención valvular; 5) la pobre protección miocárdica, secundaria a enfermedad coronaria severa o a tiempos de circulación extracorpórea prolongados, o ambos; y 6) el BAV completo en el POCC inmediato que persiste en las primeras 48 horas, sobre todo si presenta un escape infranodal.

\section{Indicación de marcapasos}

Las indicaciones de implante de MPD durante el posoperatorio son las mismas que para el paciente no sometido a cirugía. Lo controvertido y menos estudiado, dada la posible reversibilidad de estos trastornos, es el momento más adecuado para el implante. Según Socie y colaboradores ${ }^{(15)}$, aproximadamente el $42 \%$ de los BAV de alto grado mejoran en un período medio de tres días. Los autores sostienen que el $90 \%$ de los pacientes que recuperan la conducción lo hacen antes del séptimo día del POCC. Según Kim y colaboradores ${ }^{(37)}$, el desarrollo de BAVC en las primeras 24 horas del POCC valvular que se mantiene durante las 48 horas siguientes, tiene una elevada probabilidad de permanecer, por lo que aconsejan el implante de MPD antes de la primera semana. Merin y colaboradores ${ }^{(3)}$ recomiendan el implante de MPD al quinto día del POCC en pacientes que mantienen el bloqueo y fueron sometidos a SVA, o presentaban BCRI en el preoperatorio.

El implante de un MPD permite la pronta movilización, reduciendo así la morbilidad asociada con estancia prolongada en cama. La indicación del implante está dada por BAV en más del $80 \%(3,12,14,22)$ de los casos, siendo el resto por ENS (bradicardia severa sintomática y fibrilación auricular con respuesta ventricular lenta).

La Sociedad Europea de Cardiología(38), en su guía de estimulación cardíaca publicada en el año 2013, recomienda un período de espera de cinco a siete días después de la cirugía para el implante de MPD en pacientes con BAV avanzado o completo y disfunción sinusal sintomática. Sin embargo, establecen que la presencia de un ritmo de escape bajo en el BAV completo, puede acortar este tiempo, dado que es poco probable que se resuelva espontáneamente (indicación I, evidencia C).

De acuerdo con la guía publicada en 2018 por American College of Cardiology y American Heart Association $^{(39)}$, está indicado el implante de MPD en el posoperatorio de CRM o sustitución valvular 
antes del alta, cuando la ENS o el BAV se asocian con síntomas o inestabilidad hemodinámica que no se resuelven (indicación I, B). En cuanto al tiempo de espera, no hay estudios específicos, pero plantean como razonable un plazo de tres a cinco días en la SVA y cirugía tricúspide, y algo mayor, de cinco a siete días, en la sustitución valvular mitral y CRM. En todos los pacientes sometidos a cirugía cardíaca, recomiendan el implante de marcapasos epicárdico temporal (indicación I, evidencia C, para cirugía aórtica y tricúspide y IIa para cirugía valvular mitral y CRM). También establecen que se debe considerar, en pacientes sometidos a reemplazo o reparación de válvula tricúspide con alto riesgo de BAV completo posoperatorio, el implante de electrodos de estimulación epicárdica permanente en el momento de la cirugía (indicación IIa, evidencia C).

No existen recomendaciones en las guías, con respecto al BAV que se presenta luego de la cirugía valvular por endocarditis. Las guías de endocarditis ${ }^{(31)}$ se refieren al marcapasos como dispositivo vinculado a la infección, y a su extracción y oportunidad de reimplante para evitar nuevas infecciones. Establecen la necesidad de hemocultivos negativos al menos 72 horas previo al procedimiento, y si hay evidencia de infección valvular remanente, el implante debe retrasarse al menos 14 días. Además, plantean que la estimulación temporal en la endocarditis es un factor de riesgo para la infección de un dispositivo cardíaco posterior, y si es posible, debe evitarse. El uso temporal de cables de fijación activa conectados a dispositivos externos se describe como una terapia "puente" en pacientes dependientes, permitiendo la movilización precoz con un bajo riesgo de pérdida de captura.

En la endocarditis de múltiples válvulas o abscesos, complicados con BAV, la conducta más adecuada es el implante de electrodos epicárdicos bipolares durante la cirugía y, si tiene indicación, bicamerales, para lo cual es fundamental trabajar en conjunto con los cirujanos cardíacos. La estimulación epicárdica definitiva, a través de electrodos bipolares, ha demostrado mantener bajos umbrales de estimulación y excelente detección en el seguimiento en comparación con los electrodos unipolares, con mayor longevidad de la batería(40) ${ }^{(4)}$ Esta conducta terapéutica disminuye la estimulación temporal prolongada, la estadía hospitalaria y, principalmente el riesgo de nuevas infecciones endocavitarias.

\section{Morbilidad y mortalidad}

Los pacientes con trastornos de la conducción presentan una estadía hospitalaria más larga y mayor $\operatorname{morbilidad}^{(1,8,14)}$. Según Mehaffey y colaboradores ${ }^{(11)}$, el grupo con MPD duplicó la estancia en cui- dados intensivos y tuvo una internación más prolongada; también fue mayor el reingreso hospitalario en los primeros 30 días del POCC. Todo esto se reflejó en un aumento significativo del costo sanitario.

El pronóstico para el BCRD es favorable y la mayoría son transitorios. Con respecto a los trastornos de la conducción de los fascículos izquierdos, su carácter es más permanente, y se los ha correlacionado con un mayor daño miocárdico y mortalidad operatoria cuando se asocian a infartos extensos ${ }^{(6,41)}$. Algunos estudios reportaron mayor mortalidad operatoria en pacientes revascularizados que presentaron BAV completo ${ }^{(7,8)}$; sin embargo, también tuvieron una mayor incidencia de bajo gasto cardíaco e infarto de miocardio posoperatorio, por lo que el BAV no fue el único factor implicado en el aumento de la mortalidad. Una revisión publicada en 2006 sobre los trastornos de la conducción en pacientes con CRM, no encontró asociación significativa entre dichas alteraciones y la mortalidad posoperatoria, aunque como limitación, los autores reportaron que el BCRD fue el trastorno más frecuente dentro de los incluidos ${ }^{(5)}$. Bagur y colaboradores ${ }^{(14)}$ reportaron una tasa de mortalidad similar a 30 días entre los pacientes con y sin marcapasos.

Se reconoce que la estimulación desde el ápex del ventrículo derecho (VD) provoca una disincronía mecánica, que en algunos pacientes puede desencadenar remodelación ventricular con reducción de la fracción de eyección e insuficiencia cardíaca ${ }^{(42)}$. Parece razonable esperar que el implante de MPD después de la cirugía cardíaca se asocie a mayor mortalidad en el seguimiento; sin embargo, Bagur y colaboradores ${ }^{(14)}$ y Raza y colaboradores ${ }^{(43)}$ no encontraron dicha asociación. Por otro lado, Wiggins y colaboradores ${ }^{(4)}$ informaron una probable disminución en la supervivencia en aquellos pacientes con estimulación apical.

Estudios recientemente publicados por Greason y colaboradores ${ }^{(12)}$ y Mehaffey y colaboradores ${ }^{(11)}$, que incluyeron pacientes con SVA aislada o combinada, y una mediana de seguimiento de 11 años, encontraron que el implante de MPD es un factor independiente asociado con mayores tasas de mortalidad. Su efecto sobre la mortalidad a largo plazo fue mayor que otras complicaciones importantes del posoperatorio, incluidas la fibrilación auricular y la mediastinitis ${ }^{(11)}$. En el trabajo de Greason y colaboradores $^{(12)}$ se informó que $98 \%$ de los electrodos fueron implantados en el ápex del VD. Actualmente, el lugar recomendado es el septum medio del $\mathrm{VD}^{(44)}$, por asociarse a menor incidencia de disincronía. Creemos que se requerirán nuevos estudios de seguimiento longitudinal para revalorar estos hallazgos sobre la mortalidad. 
La evidencia actual sobre el uso del MPD luego de la cirugía cardíaca y la dependencia a este a largo plazo es limitada debido a la falta de un diseño estandarizado en los estudios. Durante el seguimiento se ha encontrado una mayor dependencia si la indicación del implante fue un $\mathrm{BAV}^{(3,45)}$. Según Kiehl y colaboradores ${ }^{(46)}$, la recuperación tardía de la conducción $\mathrm{AV}$ ocurre aproximadamente en $12 \%$ de los pacientes dentro de los seis meses siguientes. Entre los predictores de recuperación de la conducción, reportaron el sexo femenino y el antecedente de conducción AV transitoria en el POCC, y como predictor de dependencia el antecedente preoperatorio de trastornos de la conducción.

\section{Conclusiones}

Los trastornos del sistema de conducción son frecuentes en el POCC. Se presentan generalmente como bloqueos fasciculares y diferentes grados de $\mathrm{BAV}$, siendo en su mayoría reversibles. De 1\%-6\% de las CRM y valvulares requieren implante de MPD.

Los mecanismos fisiopatológicos implicados durante la cirugía son el trauma mecánico, el daño isquémico y la pobre protección miocárdica. La mayor incidencia de trastornos en la cirugía valvular se debe al trauma mecánico directo del sistema de conducción, secundario a la proximidad anatómica que presenta con los anillos valvulares, aórtico y tricúspide. Las indicaciones de implante de MPD no difieren de las establecidas para los pacientes sin cirugía, sin embargo, dada la posible reversibilidad de los trastornos durante el POCC, las guías realizan solo recomendaciones sobre los plazos de espera para el implante. Los predictores más frecuentemente vinculados con la persistencia de estos trastornos de la conducción son la mayor edad del paciente, las alteraciones previas de la conducción (sobre todo BCRI o BCRD), la cirugía valvular (más frecuentemente aórtica), y la evidencia de BAV completo precoz que se mantiene en las primeras 48 horas (en particular si presenta escape infranodal). Destacamos la importancia de reconocer estos predictores para identificar qué pacientes se benefician de un implante precoz de MPD durante el POCC.

Zuly Cortellezzi, https://orcid.org//0000-0003-0123-8101 Gustavo Tortajada, https://orcid.org/0000-0002-9522-8299 Romina Roif, https://orcid.org/0000-0001-6531-587X

Gonzalo Varela, https://orcid.org/0000-0002-6476-3416

Este artículo fue aceptado para su publicación por: Editor jefe Dr. Gerardo Soca.

\section{Bibliografía}

1. Gordon R, Ivanov J, Cohen G, Ralph-Edwards A. Permanent cardiac pacing after a cardiac operation: predicting the use of permanent pacemakers. Ann Thorac Surg. 1998; 6 6(5):1698-704. doi: 10. 1016/s0003-4975(98)00889-3

2. Goldman B, Hill T, Weisel R, Scully H, Mickleborough L, Pym J, et al. Permanent cardiac pacing after open-heart surgery: acquired heart disease. Pacing Clin Electrophysiol. 1984; 7(3 Pt 1):367-71. doi: 10.1111/j.1540-8159.1984.tb04919.x

3. Merin O, Ilan M, Oren A, Fink D, Deeb M, Bitran D, et al. Permanent pacemaker implantation following cardiac surgery: indications and long-term follow-up. Pacing Clin Electrophysiol. 2009; 32(1): 7-12. doi: 10.1111/j.1540-8159.2009.02170.x

4. Wiggins $\mathbf{N}$, Chong $\mathbf{D}$, Houghtaling $\mathbf{P}$, Hussein A, Saliba W, Sabik J, et al. Incidence, indications, risk factors, and survival of patients undergoing cardiac implantable electronic device implantation after open heart surgery. Europace 2017; 19(8):1335-42. doi: 10.1093/europace/euw234

5. Kumbhani D, Sharma G, Khuri S, Kirdar J. Fascicular conduction disturbances after coronary artery bypass surgery: a review with a meta-analysis of their long-term significance. J Card Surg. 2006; 21(4):428-34. doi: 10.1111/j.1540-8191.2006.00264.x

6. Bateman T, Weiss M, Czer L, Conklin C, Kass R, Stewart M, et al. Fascicular conduction disturbances and ischemic heart disease: adverse prognosis despite coronary revascularization. J Am Coll Cardiol. 1985; 5(3):632-9. doi: 10.1016/s0735-1097 (85) $80388-0$

7. Caspi J, Amar R, Elami A, Safadi T, Merin G. Frequency and significance of complete atrioventricular block after coronary artery bypass grafting. Am J Cardiol. 1989; 63(9):526-9. doi: 10.1016/00029149(89)90893-x

8. Piantá R, Ferrari A, Heck A, Ferreira D, Piccoli J, Albuquerque L, et al. Atrioventricular block in coronary artery bypass surgery: perioperative predictors and impact on mortality. Rev Bras Cir Cardiovasc. 2015 ; 30(2):164-72. doi: 10.5935/1678-9741. 20140086

9. Leyva F, Qiu T, McNulty D, Evison F, Marshall H, Gasparini M. Long-term requirement for pacemaker implantation after cardiac valve replacement surgery. Heart Rhythm 2017; 14(4):529-34. doi: 10. 1016/j.hrthm.2016.11.029

10. Limongelli G, Ducceschi V, D'Andrea A, Renzulli A, Sarubbi B, De Feo M, et al. Risk factors for pacemaker implantation following aortic valve replacement: a single centre experience. Heart 2003; 89(8):901-4. doi: 10.1136/heart.89.8.901 
11. Mehaffey J, Haywood N, Hawkins R, Kern J, Teman N, Kron I, et al. Need for permanent pacemaker after surgical aortic valve replacement reduces long-term survival. Ann Thorac Surg. 2018; 106(2):460-5. doi: 10.1016/j.athoracsur.2018.02.041

12. Greason K, Lahr B, Stulak J, Cha Y, Rea R, Schaff H, et al. Long-Term mortality effect of early pacemaker implantation after surgical aortic valve replacement. Ann Thorac Surg. 2017; 104(4):1259-64. doi: $10.1016 / j$.athoracsur.2017.01.083

13. Baraki H, Al Ahmad A, Jeng-Singh S, Saito S, Schmitto J, Fleischer B, et al. Pacemaker dependency after isolated aortic valve replacement: do conductance disorders recover over time? Interact Cardiovasc Thorac Surg. 2013; 16(4):476-81. doi: 10.1093/icvts/ivs555

14. Bagur R, Manazzoni J, Dumont É, Doyle D, Perron J, Dagenais F, et al. Permanent pacemaker implantation following isolated aortic valve replacement in a large cohort of elderly patients with severe aortic stenosis. Heart 2011; 97(20):1687-94. doi: 10.1136/heartjnl-2011-300308

15. Socie $\mathbf{P}$, Nicot $\mathbf{F}$, Baudinaud $\mathbf{P}$, Estagnasie $\mathbf{P}$, Brusset A, Squara P, et al. Frequency of recovery from complete atrioventricular block after cardiac surgery. Am J Cardiol. 2017; 120(10):1841-6. doi: 10.1016/j.amjcard.2017.07.090

16. Andreas M, Wallner S, Habertheuer A, Rath C, Schauperl M, Binder T, et al. Conventional versus rapid-deployment aortic valve replacement: a single-centre comparison between the Edwards Magna valve and its rapid-deployment successor. Interact Cardiovasc Thorac Surg. 2016; 22(6):799-805. doi: 10.1093/icvts/ivw052

17. Meimoun P, Zeghdi R, D'Attelis N, Berrebi A, Braunberger E, Deloche A, et al. Frequency, predictors, and consequences of atrioventricular block after mitral valve repair. Am J Cardiol. 2002; 89(9):1062-6. doi: 10.1016/s0002-9149(02)02276-2

18. Berdajs D, Schurr U, Wagner A, Seifert B, Turina M, Genoni M. Incidence and pathophysiology of atrioventricular block following mitral valve replacement and ring annuloplasty. Eur J Cardiothorac Surg. 2008; 34(1):55-61. doi: 10.1016/j.ejcts.2008.03.051

19. Viles-Gonzalez J, Enriquez A, Castillo J, Coffey J, Pastori L, Reddy V, et al. Incidence, predictors, and evolution of conduction disorders and atrial arrhythmias after contemporary mitral valve repair. Cardiol J. 2014; 21(5):569-75. doi: 10.5603/CJ.a2014.0016

20. Scully H, Armstrong C. Tricuspid valve replacement. Fifteen years of experience with mechanical prostheses and bioprostheses. J Thorac Cardiovasc Surg. 1995; 109(6):1035-41. doi: 10.1016/S0022-522 3(95) 70185-0
21. Jaeger F, Trohman R, Brener S, Loop F. Permanent pacing following repeat cardiac valve surgery. Am J Cardiol. 1994; 74(5):505-7. doi: 10.1016/0002-9149(94)90916-4

22. Elahi MM, Lee D, Dhannapuneni RR. Predictors of permanent pacemaker implantation during the early postoperative period after valve surgery. Tex Heart Inst J. 2006;33(4):455-7.

23. Lewis J, Webb C, Pickard S, Lehman J, Jacobsen $\mathbf{G}$. The increased need for a permanent pacemaker after reoperative cardiac surgery. J Thorac Cardiovasc Surg. 1998; 116(1):74-81. doi: 10.1016/ S0022-5223(98)70245-4

24. Anderson R, Yanni J, Boyett M, Chandler N, Dobrzynski H. The anatomy of the cardiac conduction system. Clin Anat. 2009; 22(1):99-113. doi: 10.1002/ca.20700

25. Reyes W, Tortajada G, Varela G, Cortellezzi Z. Bloqueos auriculoventriculares de primer, segundo y tercer grado. En: Sociedad Interamericana de Cardiología. Texto de Cardiología. Bogotá: Distribuna, 2019: Cap.53.

26. Futami C, Tanuma K, Tanuma Y, Saito T. The arterial blood supply of the conducting system in normal human hearts. Surg Radiol Anat. 2003; 25(1):42-9. doi: 10.1007/s00276-002-0085-7

27. Yater W, Cornell V. Heart block due to calcareous lesions of the bundle of His: review and report of a case with detailed histopathologic study. Ann Intern Med. 1935; 8(7):777-89. doi: 10.7326/0003-4819-8-7-777

28. Sellers R, Kanjuh V, Eliot R, Bonnabeau R, Edwards J, Lillehei C. Complete heart block following aortic and mitral valve replacement: anatomic basis, prevention and management. Circulation 1963; 28(4):801. doi: 10.1161/01.CIR.28.4.679

29. Fukuda T, Hawley R, Edwards J. Lesions of conduction tissue complicating aortic valvular replacement. Chest 1976; 69(5):605-14. doi: 10.1378/chest. 69.5.605

30. Totaro P, Calamai G, Montesi G, Barzaghi C, Vaccari M. Continuous suture technique and impairment of the atrioventricular conduction after aortic valve replacement. J Card Surg. 2000; 15(6):418-22. doi: $10.1111 / \mathrm{j} .1540-8191.2000 . t b 01302 . x$

31. Habib G, Lancellotti P, Antunes M, Bongiorni M, Casalta J, Del Zotti F, et al. 2015 ESC Guidelines for the management of infective endocarditis: The Task Force for the Management of Infective Endocarditis of the European Society of Cardiology (ESC). Endorsed by: European Association for Cardio-Thoracic Surgery (EACTS), the European Association of Nuclear Medicine (EANM). Eur Heart J. 2015; 36(44):3075-128. doi: 10.1093/eurheartj/ehv319 
32. Matthews I, Fazal I, Bates M, Turley A. In patients undergoing aortic valve replacement, what factors predict the requirement for permanent pacemaker implantation? Interact Cardiovasc Thorac Surg. 2011; 12(3):475-9. doi: 10.1510/icvts.2010. 254607

33. Erdogan H, Kayalar N, Ardal H, Omeroglu S, Kirali K, Guler M, et al. Risk factors for requirement of permanent pacemaker implantation after aortic valve replacement. J Card Surg. 2006; 21(3):211-5; discussion 216-7. doi: 10.1111/j.1540-8191.2006.00216.x

34. Huynh H, Dalloul G, Ghanbari H, Burke P, David M, Daccarett M, et al. Permanent pacemaker implantation following aortic valve replacement: current prevalence and clinical predictors. Pacing Clin Electrophysiol. 2009; 32(12):1520-5. doi: 10.1111/j. $1540-8159.2009 .02552 . x$

35. Friedman H, Zaman Q, Haft J, Melendez S. Assessment of atrioventricular conduction in aortic valve disease. Br Heart J. 1978; 40(8):911-7. doi: 10.1136/hrt.40.8.911

36. Koplan B, Stevenson W, Epstein L, Aranki S, Maisel W. Development and validation of a simple risk score to predict the need for permanent pacing after cardiac valve surgery. J Am Coll Cardiol. 2003; 41(5):795-801. doi: 10.1016/s0735-1097(02)02926-1

37. Kim M, Deeb G, Eagle K, Bruckman D, Pelosi F, Oral H, et al. Complete atrioventricular block after valvular heart surgery and the timing of pacemaker implantation. Am J Cardiol. 2001; 87(5):649-51, A10. doi: 10.1016/s0002-9149(00)01448-x

38. Brignole M, Auricchio A, Baron-Esquivias G, Bordachar P, Boriani G, Breithardt O, et al. 2013 ESC Guidelines on cardiac pacing and cardiac resynchronization therapy: the Task Force on cardiac pacing and resynchronization therapy of the $\mathrm{Eu}-$ ropean Society of Cardiology (ESC). Developed in collaboration with the European Heart Rhythm Association (EHRA). Eur Heart J. 2013; 34(29):2281-329. doi: 10.1093/eurheartj/eht150

39. Kusumoto F, Schoenfeld M, Barrett C, Edgerton J, Ellenbogen K, Gold M, et al. 2018
ACC/AHA/HRS Guideline on the evaluation and management of patients with bradycardia and cardiac conduction delay: a report of the American College of Cardiology/American Heart Association Task Force on Clinical Practice Guidelines and the Heart Rhythm Society. Circulation 2019; 140(8):e382e482. doi: 10.1161/CIR.0000000000000628

40. Tomaske M, Gerritse B, Kretzers L, Pretre R, Dodge-Khatami A, Rahn M, et al. A 12-year experience of bipolar steroid-eluting epicardial pacing leads in children. Ann Thorac Surg. 2008; 85(5):1704-11. doi: 10.1016/j.athoracsur.2008.02.016

41. Zeldis S, Morganroth J, Horowitz L, Michelson E, Josephson M, Lozner E, et al. Fascicular conduction disturbances after coronary bypass surgery. Am J Cardiol. 1978; 41(5):860-4. doi: 10.1016/ 0002-9149(78)90725-7

42. Tops L, Schalij M, Bax J. The effects of right ventricular apical pacing on ventricular function and dyssynchrony implications for therapy. J Am Coll Cardiol. 2009; 54(9):764-76. doi: 10.1016/j.jacc.2009.06.006

43. Raza S, Li J, John R, Chen L, Tholakanahalli V, Mbai M, et al. Long-term mortality and pacing outcomes of patients with permanent pacemaker implantation after cardiac surgery. Pacing Clin Electrophysiol. 2011; 34(3):331-8. doi: 10.1111/j.15408159.2010.02972.x

44. Cano O, Sancho-Tello M, Osca J, Olague J. Estimulación desde el tracto de salida del ventrículo derecho. Cuad Estimul Card. 2011; 4(10):27-36.

45. Steyers C, Khera R, Bhave P. Pacemaker dependency after cardiac surgery: a systematic review of current evidence. PLoS One. 2015; 10(10):e0140340. doi: 10.1371/journal.pone.0140340

46. Kiehl E, Makki T, Matar R, Johnston D, Rickard J, Tarakji K, et al. Incidence and predictors of late atrioventricular conduction recovery among patients requiring permanent pacemaker for complete heart block after cardiac surgery. Heart Rhythm 2017; 14(12):1786-92. doi: 10.1016/j.hrthm.2017. 08.009 